\title{
On the Principle of Invariant Imbedding and Neutron Transport Theory I-One-Dimensional Case
}

\section{RICHARD BELLMAN, ROBERT KALABA \& G. MILTON WING*}

1. Introduction. In a series of notes, [1], [2], [3], we have sketched some applications of the Principle of Invariant Imbedding, and the associated functional equation approach, to an important class of problems in radiative transfer, a field pioneered and extensively cultivated by Ambarzumian and CHandraSEKHAR, [4], and to a class of problems in neutron diffusion theory.

In this paper we wish to develop in some detail the techniques presented in the third note above, [3]. We shall consider a one-dimensional version of the reflection and transmission of neutrons, and the phenomenon of critical mass. Although we shall consider the effects of fission, capture and forward and backward scattering, we shall not take into account collisions between neutrons.

Our basic model will be stochastic, with standard transport theory derived on the basis of expected values. As the explicit form of the higher moments indicates, an expected value theory yields a quite inadequate view of the distribution of reflected and transmitted neutrons in the neighborhood of critical mass.

We shall present some numerical results describing these distributions obtained from the solution of forty simultaneous nonlinear differential equations. Problems of this nature, once so formidable, can be done in a routine fashion with modern digital computers.

In particular, we would like to emphasize the fact that the determination of critical length in the one-dimensional model, and critical dimensions in the general case, is made to depend upon the solution of nonlinear differential equations with one-point conditions, initial conditions, rather than upon the solution of two-point boundary value problems which involve characteristic values.

In subsequent publications, we will apply the same techniques to multidimensional models, and discuss in detail the connection between the various modes of approach.

*Work performed in part under the auspices of the U. S. Atomic Energy Commission. 
2. Invariant Imbedding and Neutron Diffusion. Let us now discuss the conceptual features of the technique we shall employ to formulate reflection and transmission questions. The method we use is entirely different from the classical approach based on a Boltzmans, or transport, equation. That approach rests upon the concept of a neutron density, and is thus an expected value theory, with the usual advantages and disadvantages.

Consider a neutron, which can proceed only in a forward or backward fashion, entering a $\operatorname{rod}$ at $A$ as below.

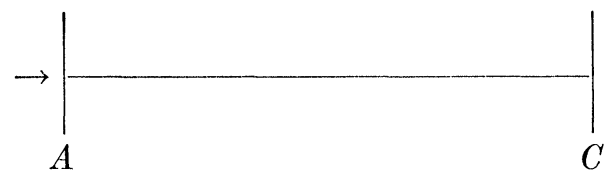

Figure 1.

Suppose that the neutron has penetrated to the intermediate point $B$.

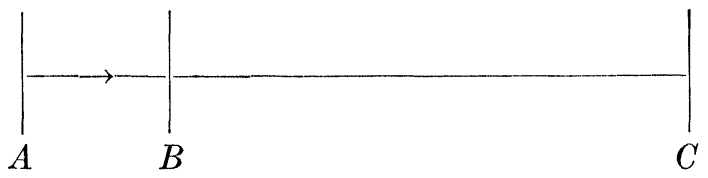

Figure 2.

In order to treat the reflection or transmission problem for the original rod $A C$ we see that we must consider a similar problem for the $\operatorname{rod} B C$.

This realization that the specific problem pertaining to reflection and transmission properties of a fixed rod $A C$ can be subsumed within the general problem of solving similar problems for rods of all sizes, and, furthermore, that these problems are interconnected, is a particular consequence of what we have called the Principle of Invariant Imbedding, [1].

In order to treat our problems analytically, it remains only to translate the above ideas into mathematical symbolism.

3. The Underlying Physical Model. Consider a homogeneous one-dimensional rod taken to consist of nuclei with which neutrons moving to the right or to the left may collide. We shall suppose that the collision of a neutron with a nucleus produces one of the following four results:

(1) 1. Production of two neutrons, replacing the old neutron (fission).

2. Scattering of the neutron in the direction in which it has been travelling (forward scattering).

3. Scattering of the neutron resulting in a reversal of direction (backward scattering).

4. Disappearance of the neutron (capture). 
We shall assume that there is no energy or velocity dependence and, furthermore, that collision between neutrons can be disregarded. These more realistic cases will be discussed at a later date.

To simplify the bookkeeping, we shall assume that when fission occurs, one offspring proceeds to the right and the other to the left. The methods we present can handle equally well the situation in which there is a probability distribution for the results of fission, and, in addition, the case where there is a probability distribution governing the number of neutrons produced by fission. Furthermore, the case in which the probabilities of collision of various types depend upon the position in the rod may be dealt with by the same procedure.

The rod is taken to be in a vacuum which ensures that the neutrons which escape from either end are lost. In other words, once a neutron has emerged from either end of the rod, it exerts no further influence upon subsequent reflection or transmission of neutrons within the rod.

Let us define the following elementary probabilities governing the four types of collisions described above:

(2) $a \Delta=$ the probability of a fission collision in an infinitesimal length $\Delta$.

$b \Delta=$ the probability of a forward scattering collision in an infinitesimal length $\Delta$.

$c \Delta=$ the probability of a backward scattering collision in an infinitesimal length $\Delta$.

$f \Delta=$ the probability of a capture collision in an infinitesimal length $\Delta$.

Furthermore, set $\pi=a+b+c+f$.

The notation above is the usual shorthand for more precise statements in which $a \Delta$ is replaced by $(a+o(1)) \Delta$ where the $o(1)$ refers to a term which approaches zero as $\Delta \rightarrow 0$.

We wish to determine what happens when a neutron is introduced into the system from one end of the rod, say the left end. This neutron will be called a source neutron.

Let us now define the basic functions we shall discuss:

(3) $p_{n}(x)=$ the probability that exactly $n$ neutrons emerge over all time from the left end of a homogeneous rod of length $x$ as a result of one neutron entering at the left end at time zero.

We shall call $p_{n}(x)$ the probability that $n$ neutrons are reflected from the rod as the result of the source neutron. Further, let

(4) $q_{n}(x)=$ the probability that exactly $n$ neutrons emerge over all time from the right end of a homogeneous rod of length $x$ as a result of one neutron entering at the left end at time zero.

We shall call $q_{n}(x)$ the probability that $n$ neutrons are transmitted through the rod as a result of the source neutron.

The function which at first sight might seem more natural, $p_{n}(x, t)$, the prob- 
ability that exactly $n$ neutrons emerge between 0 and $t$ from the left end of a homogeneous rod of length $x$ as a result of one neutron entering at the left end at time zero, is much more difficult to treat. It requires a separate investigation which we shall present at a future date.

4. The Differential Equation for $p_{n}(x)$. In order to obtain a system of differential equations governing the sequence $\left\{p_{n}(x)\right\}$, we consider the relation between $p_{n}(x+\Delta)$ and $p_{n}(x)$, using the ideas outlined above in $\$ 2$.

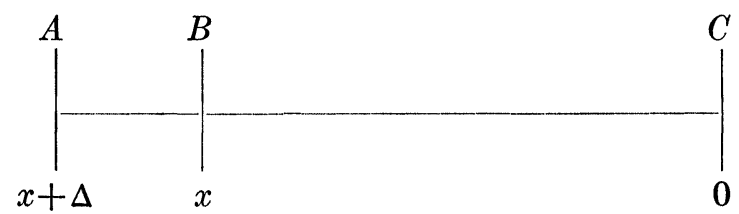

Figure 3.

Let us begin with the simplest case, $p_{0}(x)$. The probability that no neutrons are reflected from a rod of length $x+\Delta$ may be calculated as follows. A source neutron starting at $A$ may pass through $A B$ without colliding, thus becoming a source neutron for $B C$. If, as a consequence of this source neutron, no neutrons are reflected from the remaining rod of length $x$, then none are reflected from the original rod of length $x+\Delta$. If, on the other hand, a neutron passing through $A B$ results in precisely one reflected neutron from $B C$, there is still the possibility that this neutron may be captured in $A B$, or scattered backward in $A B$. In this last case, it is once again a source neutron for $B C$. Finally, a capture collision in $A B$, or a forward scattering collision, followed by no reflection from $B C$, will yield contributions to $p_{0}(x)$. It is easy to see that all other events have probabilities of order $\Delta^{2}$ for small $\Delta$. Consequently, they can be neglected in the derivation of the differential equation for $p_{0}(x)$.

Using the probabilities defined in the previous section, and writing out the probabilities associated with the foregoing eventualities, we obtain the recurrence relation

$$
\begin{gathered}
p_{0}(x+\Delta)=(1-\pi \Delta) p_{0}(x)+(1-\pi \Delta) p_{1}(x) f \Delta \\
\quad+(1-\pi \Delta) p_{1}(x)(c \Delta) p_{0}(x)+f \Delta+b \Delta p_{0}(x)+O\left(\Delta^{2}\right) \\
=(1-\pi \Delta) p_{0}(x)+f \Delta p_{1}(x)+c \Delta p_{1}(x) p_{0}(x) \\
\quad+f \Delta+b \Delta p_{0}(x)+O\left(\Delta^{2}\right) .
\end{gathered}
$$

Letting $\Delta \rightarrow 0$, the differential equation for $p_{0}(x)$ is found to be

$$
p_{0}^{\prime}(x)=-(a+c+f) p_{0}(x)+f\left(1+p_{1}(x)\right)+c p_{0}(x) p_{1}(x) .
$$

In order to obtain the corresponding result for $p_{n}(x)$, we must take account of the possibility of a neutron passing through $A B$ without collision, causing $k$ neutrons to be reflected from $B C$, one of which has a fission collision in $A B$, 
resulting in $n-k$ additional reflected neutrons from $B C$. Enumerating the cases, we obtain the results

$$
\begin{aligned}
p_{n}(x+\Delta)=(1-\pi \Delta)[ & p_{n}(x)(1-\pi \Delta)^{n}+a \Delta \sum_{k=0}^{n} k p_{k}(x) p_{n-k}(x) \\
& +n(b \Delta) p_{n}(x)+c \Delta \sum_{k=0}^{n} k p_{k}(x) p_{n-k+1}(x) \\
& \left.+(n+1)(f \Delta) p_{n+1}(x)+(n+1)(c \Delta) p_{n+1}(x) p_{0}(x)\right] \\
& +a \Delta p_{n-1}(x)+b \Delta p_{n}(x)+c \Delta \delta_{1 n}+O\left(\Delta^{2}\right) .
\end{aligned}
$$

Here $\delta_{1 n}$ is the Kronecker symbol.

Let us note that the reason for the comparative simplicity of this expression is the fact that only one of the $k$ particles, or $n$ particles, can collide in an infinitesimal interval of length $\Delta$, to terms in $\Delta^{2}$.

The resultant differential equation is

$$
\begin{aligned}
p_{n}^{\prime}(x)=-( & +1) h p_{n}(x)+\sum_{k=0}^{n} k p_{k}(x)\left[a p_{n-k}(x)+c p_{n-k+1}(x)\right] \\
& +c(n+1) p_{n+1}(x) p_{0}(x)+f(n+1) p_{n+1}(x)+a p_{n-1}(x)+c \delta_{1 n},
\end{aligned}
$$$$
\text { where } h=a+c+f \text {. }
$$

It is easy to see that the initial conditions are

$$
p_{0}(0)=1, \quad p_{n}(0)=0, \quad n>1 .
$$

5. Discussion. It remains to analyze this system of nonlinear differential equations. As we shall see, the generating function technique works very nicely, and we shall obtain a relatively simple partial differential equation for the generating function. From this we can obtain simple equations for the various moments of the distribution. The critical length will be determined from the explicit representation for the first moment.

6. Some Computational Results. Using the nonlinear system of differential equations appearing in (4.4), we had the solutions computed by two means in order to obtain some idea of what the distributions looked like as the critical length was approached. For simplicity, we have considered the case $a=1$, $b=c=f=0$.

The first method used a desk analogue computer and was prepared by M. Chiшaki. The second method used a digital computer, the Johnniac, and was prepared by Marvin Shapiro.

Although in the second case we integrated forty simultaneous differential equations of nonlinear type, we came nowhere near the capacity of the machine. It would have been as easy to have considered a system involving two hundred simultaneous nonlinear equations. This is an important point to stress since the 
application of the methods we present here to more realistic situations will involve computational problems of this magnitude in the determination of critical mass or critical radius.

Some representative curves are given below.

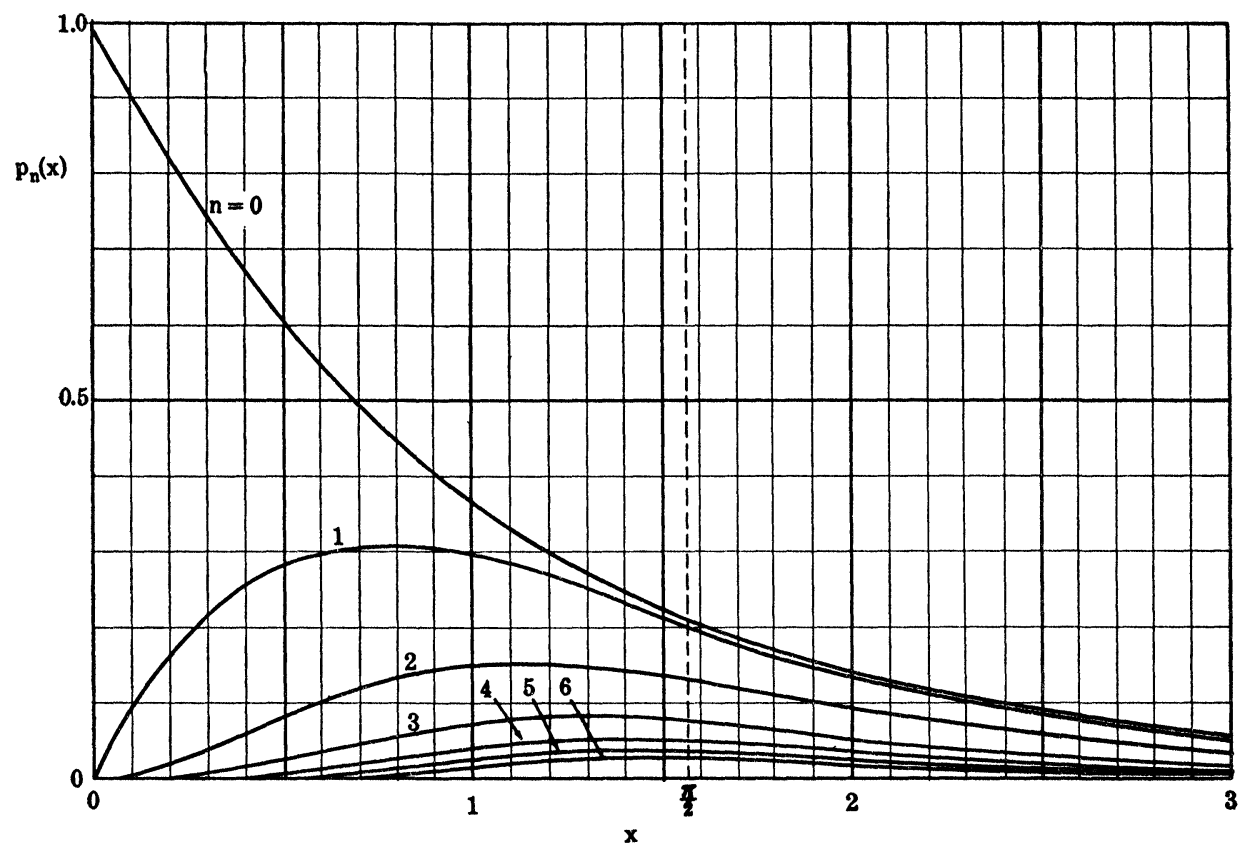

Figure 4.

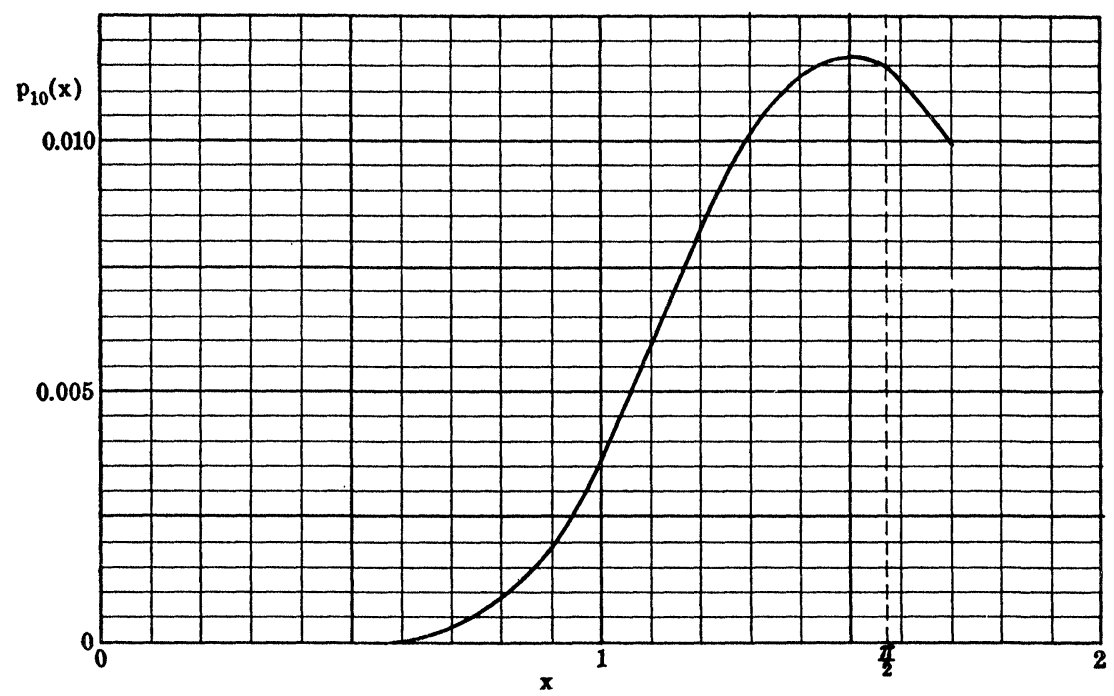

Figure 5. 


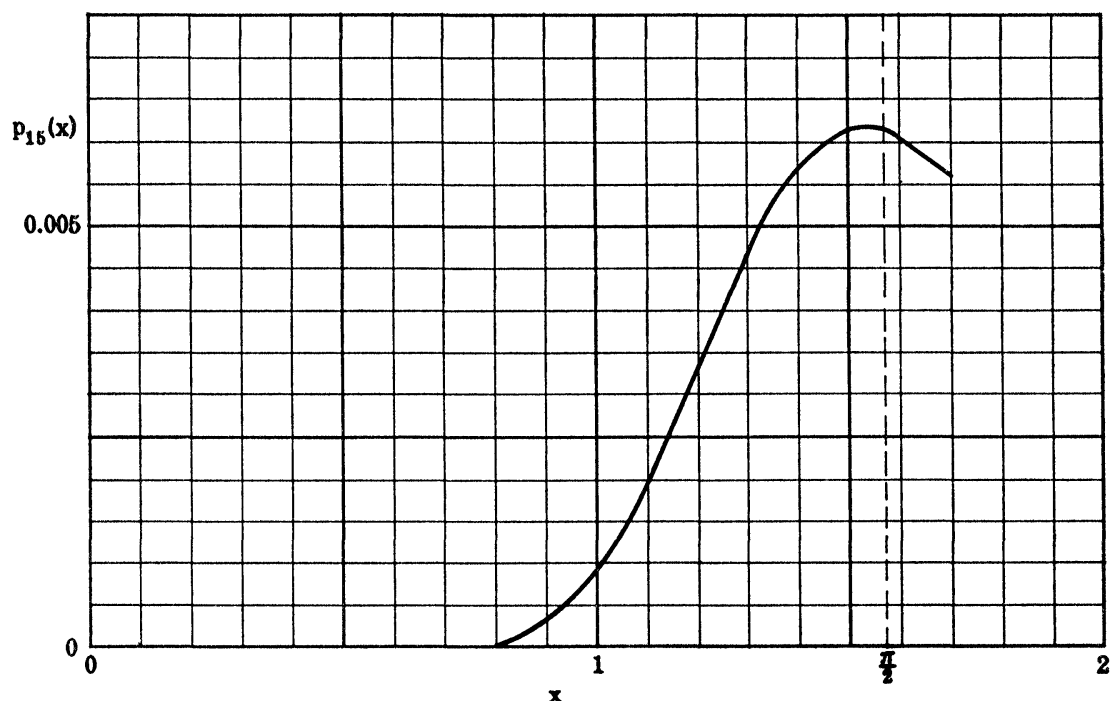

Figure 6.

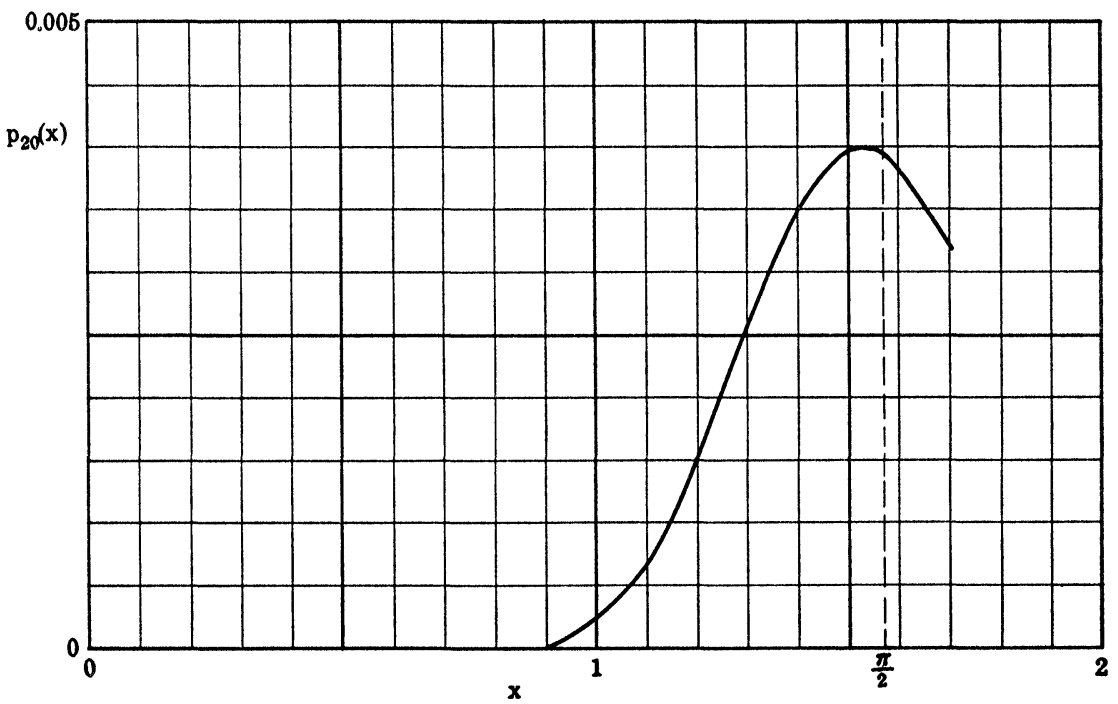

Figure 7.

7. The Generating Function. Let us now study the generating function, $u(x, t)$, defined by the relation

(1)

$$
u(x, t)=\sum_{n=0}^{\infty} p_{n}(x) t^{n}
$$


The equations in (4.4) yield, in the usual fashion, the quasilinear partial differential equation

$$
u_{x}=f u_{t}-h\left(u+t u_{t}\right)+a t u\left\{1+u_{t}\right\}+c u u_{t}+c t+f,
$$

with the initial and boundary conditions

$$
u(x, 1)=1, \quad u(0, t)=1, \quad u(x, 0)=p_{0}(x) .
$$

The first of these is the statement

$$
\sum_{k=0}^{\infty} p_{k}(x)=1
$$

while the second is obtained from (4.5). The third is not usable in any easy way in this general case, since $p_{0}(x)$ cannot be determined independently of the remaining $p_{k}(x)$.

It should be noted that the probability of forward scattering, $b$, does not appear anywhere in the above equations, and hence does not influence the solution. The concept has been introduced for the sake of completeness, since forward scattering plays an important role in more complicated processes where changes in neutron velocity are considered.

8. The Transmission Probabilities. Let us now consider the sequence $\left\{q_{n}(x)\right\}$. Referring to the following figure

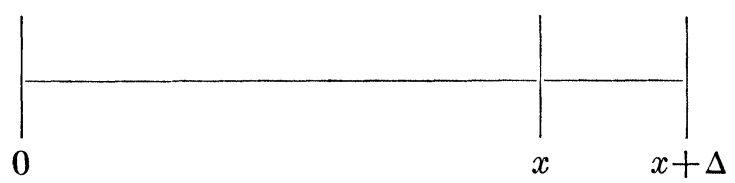

Figure 8

and employing the same reasoning, we obtain the following system of differential equations

$$
\begin{aligned}
q_{n}^{\prime}(x)=-n h q_{n}(x) & +a \sum_{k=0}^{n} k q_{k}(x) p_{n-k}(x) \\
& +c \sum_{k=0}^{n+1} k q_{k}(x) p_{n+1-k}(x)+(n+1) f q_{n+1}(x), \quad n \geqq 0,
\end{aligned}
$$

with the initial conditions

$$
q_{0}(0)=1, \quad q_{n}(0)=0, \quad n>0 .
$$

Observe that to determine the $q_{n}(x)$ we must first determine the sequence $\left\{p_{k}(x)\right\}$. 
9. The First Moment. Let us now consider the first moment

$$
E(x)=\sum_{n-1}^{\infty} n p_{n}(x)=u_{t}(x, 1) .
$$

This represents the expected number of neutrons reflected by the rod over all time. From equation (7.2) we obtain

$$
u_{t}=\frac{u_{x}+h u-a t u-c t-f}{f-h t+a t u+c u} .
$$

Substituting $t=1$ in the right-hand side of (2), we obtain an indeterminate form. Using l'Hospital's rule, we obtain the result

$$
u_{t}(x, 1)=\lim _{t \rightarrow 1}\left\{\frac{u_{x t}+h u_{t}-a u-a t u_{t}-c}{-h+a u+a t u_{t}+c u_{t}}\right\},
$$

and thus the equation

$$
E(x)=\frac{E^{\prime}(x)+(c+f) E(x)-(a+c)}{(a+c) E(x)-(c+f)} .
$$

The boundary condition

$$
E(0)=0
$$

follows from (4.5).

This first order nonlinear differential equation is readily solved either by noting that it is a Riccati equation, and hence reducible to a linear second order equation, or by a direct separation of variables.

We have, with $\mu=(c+f) /(c+a)$,

$$
E(x)=\frac{\tan \left[(a+c) \sqrt{1-\mu^{2}} x\right]}{\mu \tan \left[(a+c) \sqrt{1-\mu^{2}} x\right]+\sqrt{1-\mu^{2}}}, \quad 0 \leqq \mu<1,
$$

(6) $\quad E(x)=\frac{(a+c) x}{1+(a+c) x}, \quad \mu=1$,

$$
E(x)=\frac{\tanh \left[(a+c) \sqrt{\mu^{2}-1} x\right]}{\mu \tanh \left[(a+c) \sqrt{\mu^{2}-1 x}\right]+\sqrt{\mu^{2}-1}}, \quad \mu>1 .
$$

10. Discussion. The method of generating functions is, as we have seen, a very convenient tool for determining the moments of the distribution. We shall use it to determine the second moment in a subsequent section.

In view of the formal techniques we have employed, it is important to mention that the differential equation for $E(x)$ can be obtained directly from (4.4) by multiplying both sides of the equation by $n$ and summing over $n$. Not only is the algebraic manipulation tedious, but the result is quite unexpected from this approach, since, at first sight, the second moment seems to occur. 
11. Critical Mass. Let us now see if we can introduce the concept of a critical mass, in this case a critical length. We look for the first value of $x, x_{c}$, for which $E(x)$ becomes infinite. From the explicit expressions given in (9.6), we see that for $0 \leqq \mu<1$, a critical length is determined by the equation

$$
\mu \tan \left[(a+c) \sqrt{1-\mu^{2}} x\right]+\sqrt{1-\mu^{2}}=0 .
$$

If $\mu \geqq 1$, no critical length exists.

Let us analyze the physical meaning of these results. When $0 \leqq \mu<1$, the fission process dominates the capture process. Hence we anticipate the possibility of a critical length.

Observe that, for $x>x_{c}$, the first equation for $E(x)$ in (9.6) yields a negative expectation. The meaning of this absurdity is that the manipulations yielding this equation are invalid for $x>x_{c}$.

If $\mu>1$, capture dominates fission. Referring to (9.6), we see that $E(x)<1$, regardless of the length of the rod. When capture and fission are equally likely, $\mu=1$, we see that $E(x) \rightarrow 1$ as $x \rightarrow \infty$.

12. Simplification. In the remainder of the paper, we shall deal only with the special case $c=f=0$, which means that only fission collisions occur. This simplified model is of interest in itself, and the algebra is considerably reduced.

13. Second Moment. Let us now discuss the second moment in the modified form

$$
S(x)=\sum_{n=2}^{\infty} n(n-1) p_{n}(x)=u_{t t}(x, 1) .
$$

Under the simplifying assumptions noted in $\$ 12$, the equation for the generating function is

$$
0=-u_{x}+a u(t-1)+a t u_{t}(u-1) .
$$

Differentiation with respect to $t$, followed by some manipulation, yields

$$
u_{t t}=\frac{u_{x t}-a u_{t}(u-1)-a u-a u_{t}(u-1)-a t u_{t}^{2}}{a t(u-1)} .
$$

Use of l'Hospital's rule gives

$$
\begin{aligned}
S(x) & =\lim _{t \rightarrow 1-}\left[\frac{u_{x t t}-a u_{t t}(t-1)-2 a u_{t}-a u_{t t}(u-1)-2 a u_{t}^{2}-2 a t u_{t} u_{t t}}{a(u-1)+a t u_{t}}\right] \\
& =\frac{S^{\prime}(x)-2 a E(x)-2 a E^{2}(x)-2 a E(x) S(x)}{a E(x)} .
\end{aligned}
$$

Consequently $S(x)$ satisfies the equation

$$
S^{\prime}(x)-3 a E(x) S(x)=2 a E(x)+2 a E^{2}(x),
$$


with $S(0)=0$. In the case being considered

$$
E(x)=\tan a x,
$$

which yields

$$
S(x)=-1+2 \tan ^{3} a x+\sec a x .
$$

Thus the standard deviation of the distribution is given by

$$
\begin{aligned}
G(x) & =\sqrt{\sum_{n=0}^{\infty} n^{2} p_{n}(x)-\left\{\sum_{n=0}^{\infty} n p_{n}(x)\right\}^{2}}=\sqrt{S(x)+E(x)-E^{2}(x)} \\
& =\sqrt{2 \tan ^{3} a x+\sec a x-\sec ^{2} a x} \sim \sqrt{2}(\tan a x)^{\frac{3}{2}}
\end{aligned}
$$

as $x \rightarrow x_{c}=\pi / 2 a$.

It is clear then that the expected number of neutrons is a highly unreliable measure of the probability distribution in the neighborhood of the critical length.

14. Source Interior to Rod. So far we have assumed that the source neutron emanated from without the rod. Let us now employ the foregoing techniques to determine transmission probabilities when the source is initially inside the rod.

As we shall see, the critical length is precisely the same as before. While this is only established under the simplifying assumption that only fission occurs, a similar result may be derived for the general case.

Let the rod extend from $-x$ to $y$, with a source of one neutron moving to the right located at the origin:

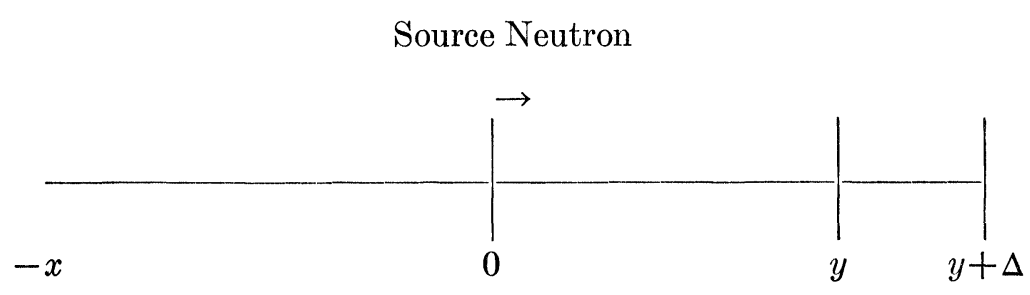

Figure 9

Let us introduce the probabilities

$$
\begin{aligned}
r_{n}(y)= & \text { the probability that exactly } n \text { neutrons emerge over all time } \\
& \text { at } y \text { as a result of a source neutron of the above type introduced } \\
& \text { at the origin at time zero. } \\
p_{n}(x+y)= & \text { the probability that exactly } n \text { neutrons emerge at } y \text { over all } \\
& \text { time as a result of a source neutron moving to the left at } y \\
& \text { at time zero. }
\end{aligned}
$$

The functions $p_{n}$ are precisely those we have encountered above. 
Proceeding as in the previous sections, we obtain the recurrence relation

$$
r_{n}(y+\Delta)=r_{n}(y)(1-a \Delta)^{n}+\sum_{k=1}^{n} r_{k}(y)(k a \Delta)(1-a \Delta)^{k-1} p_{n-k}(x+y),
$$

for $y>0, n>0$, with

$$
r_{0}(y)=0 \text {. }
$$

From this we obtain, in the limit as $\Delta \rightarrow 0$, the differential system

$$
r_{n}^{\prime}(y)=-n a r_{n}(y)+a \sum_{k=1}^{n} k r_{k}(y) p_{n-k}(x+y),
$$

$n>0, y \geqq 0$, with the boundary conditions

$$
r_{1}(0)=1, \quad r_{n}(0)=0, \quad n>1 .
$$

15. The Generating Function. Introducing the generating function

$$
w(y, t)=\sum_{n=0}^{\infty} r_{n}(y) t^{n}
$$

it is easily seen that

$$
w_{y}=\operatorname{atw}_{t}\left\{\sum_{n=0}^{\infty} p_{n}(x+y) t^{n}-1\right\},
$$

with the boundary conditions

$$
w(0, t)=t, \quad w(y, 0)=0, \quad w(y, 1)=1 .
$$

The expected number of neutrons transmitted is given by

$$
Z(y)=\sum_{n=0}^{\infty} n r_{n}(y)=w_{t}(y, 1) .
$$

Following the method used above, we obtain the differential equation

$$
a Z(y)=\frac{Z^{\prime}(y)}{E(x+y)}=\frac{Z^{\prime}(y)}{\tan a(x+y)} .
$$

It follows that

$$
Z(y)=\frac{\cos a x}{\cos a(x+y)}
$$

an expression which is valid for $x+y<\pi / 2 a, x, y \geqq 0$. The critical length is $\pi / 2 a$, in agreement with the result obtained when the source neutron was introduced externally. This result can be predicted on probabilistic grounds.

16. Comparison with Classical Results. The problem we have considered may, of course, be treated by means of classical transport theory. 
Let us see what this method yields for the case where the source neutron is initially on the left end of the rod. Let $u_{+}(x)$ be the density of neutrons moving to the right at $x$ and $u_{-}(x)$ the density of neutrons moving to the left at $x$. Let $0 \leqq x \leqq l$, where $l$ is the length of the rod.

Following the treatment in [4], p. 55, we obtain the equations

$$
\begin{aligned}
& \frac{d u_{+}}{d x}+a u_{+}=a\left(u_{+}+u_{-}\right), \\
& \frac{-d u_{-}}{d x}+a u_{-}=a\left(u_{+}+u_{-}\right) .
\end{aligned}
$$

The assumption of unit source at the left end of the rod yields the boundary condition

$$
u_{+}(0)=1 \text {. }
$$

Since no neutrons may re-enter the rod at the right end, we must have

$$
u_{-}(l)=0 \text {. }
$$

This system of equations has the solution

$$
\begin{aligned}
& u_{+}(x)=\frac{\cos a(l-x)}{\cos a l}, \\
& u_{-}(x)=\frac{\sin a(l-x)}{\cos a l} .
\end{aligned}
$$

In particular,

$$
u_{-}(0)=\tan a l=E(l) .
$$

Thus the outgoing neutron density in the classical deterministic treatment is merely the mean of the distribution in the probabilistic treatment.

In this connection, cf. $\$ 13$.

17. Outstanding Problems and Conjectures. Examining the calculations described in $\$ 6$, we are led to some interesting conjectures which seem quite difficult to verify.

For the case where only fission collisions occur, it seems highly likely that $p_{n}(x)<p_{n-1}(x), n \geqq 1, x>0$. This should be derivable directly from the nonlinear system of differential equations given in (4.4), but so far this has not been done.

An examination of the maxima over $x$ of the curves for $p_{n}(x)$ gives rise to some further conjectures. Let the maximum of $p_{n}(x)$, assumed to be uni-modal, occur at $x=y_{n}$. Then the calculations seem to show that

$$
\frac{\pi}{2 a}>y_{n}>y_{n-1}>0
$$


and that

$$
\lim _{n \rightarrow \infty} y_{n}=\frac{\pi}{2 a}
$$

the critical length.

This last conjecture we expect to demonstrate by means of an explicit solution of the quasi-linear partial differential equation for $u$ via characteristics. In this way we hope to obtain the asymptotic form of $p_{n}(x)$ as $n \rightarrow \infty$, and so verify (2).

18. Further Work in Progress. As indicated in our note, [3], and in the introduction, we intend to pursue the application of these techniques in a number of directions. In particular, we are examining the many-velocity case for one dimension, the time-dependent case, and higher dimensions and more complicated geometries.

\section{REFERENCES}

[1] R. Bellman \& R. Kalaba, On the principle of Invariant Imbedding and Propagation through Inhomogeneous Media, Proc. Nat. Acad. Sci. USA, 42 (1956), pp. 629-632.

[2] R. Bellman \& R. Kalaba, On the Principle of Invariant Imbedding and Diffuse Reflection from Cylindrical Regions, Proc. Nat. Acad. Sci. USA, 43 (1957), pp. 514-517.

[3] R. Bellman, R. Kalaba \& G. M. Wing, On the Principle of Invariant Imbedding and One-Dimensional Neutron Multiplication, Proc. Nat. Acad. Sci. USA, 43 (1957), pp. $517-520$.

[4] S. Chandrasekhar, Radiative Transfer, Oxford, 1950.

The Rand Corporation

Santa Monica, California and

The Los Alamos Scientific Laboratory

Los Alamos, New Mexico 